\title{
Germanica
}

\section{La chronique des événements courants de Peter Handke : une chronique des images}

Handkes Chronik der laufenden Ereignisse: eine Chronik der laufenden Bilder

\section{Arlette Camion}

\section{OpenEdition}

\section{Journals}

Édition électronique

URL : http://journals.openedition.org/germanica/2224

DOI : $10.4000 /$ germanica.2224

ISSN : 2107-0784

Éditeur

Université de Lille

Édition imprimée

Date de publication : 1 décembre 1994

Pagination : 177-187

ISSN : 0984-2632

Référence électronique

Arlette Camion, «La chronique des événements courants de Peter Handke : une chronique des images », Germanica [En ligne], 14 | 1994, mis en ligne le 30 septembre 2013, consulté le 06 octobre 2020. URL: http://journals.openedition.org/germanica/2224 ; DOI : https://doi.org/10.4000/germanica.2224

Ce document a été généré automatiquement le 6 octobre 2020.

(c) Tous droits réservés 


\section{La chronique des événements courants de Peter Handke : une chronique des images}

Handkes Chronik der laufenden Ereignisse: eine Chronik der laufenden Bilder Arlette Camion

1 En 1968, Peter Handke est un jeune représentant de l'avant-garde et c'est à ce titre que la télévision allemande lui propose d'écrire le scénario d'un téléfilm. Le manuscrit terminé, les difficultés commencent: aucun metteur en scène ne veut se charger du tournage, si bien que Handke finit par en prendre la direction et se heurte bientôt à l'incompréhension de l'équipe, aux problèmes financiers, à ses propres hésitations. Le film est toutefois réalisé et sera diffusé sur le Westdeutscher Rundfunk en mai 1971. Handke s'explique sur son projet dans une interview donnée peu après :

Ich wollte an sich eine Allegorie schreiben vom Leben in der Bundesrepublik im Jahre 1969 und in den vorangegangenen Jahren. Die Form dafür war die einer Chronik. Ich wollte zeigen, wie die Fernsehbilder das Bild von der Geschichte, von der Zeit, in der man lebt, beeinflußen. Es gibt eine sowjetische Untergrundzeitung, die heißt «Chronik der laufenden Ereignisse», und ich fand das einen sehr schönen Titel $^{1}$.

2 Mi-critique, mi-récit, le film de Handke est délibérément hybride. Il veut d'une part raconter l'histoire de deux hommes, Spade et Beaumont, qui débarquent dans une ville (i.e. la République Fédérale prise dans les événements de mai 1968 et des mois suivants). Spade et Beaumont sont des héros empruntés au Faucon Maltais et à La clef de verre de Dashiel Hammett, que Handke avait d'abord pensé adapter. Ce premier niveau constitue une diégèse floue, parfois à peine discernable. Car elle est prise dans ce que Handke veut d'autre part raconter : une simple tranche de télévision. En renonçant à la pure narration filmique, Handke porte son attention sur la nature et la fonction du phénomène télévisuel. Mais en n'y renonçant pas tout à fait, car une intrigue continue de courir en filigrane tout le long de son film, il rend justice à la fonction narrative de toute image-mouvement. Cette position ambiguë lui permet de montrer l'ambiguïté que produit et masque à la fois le petit écran. Il n'y a rien de didactique dans son projet, 
la critique n'est pas ici distance, mais au contraire observation d'un phénomène « en temps réel». Voilà pourquoi la "chronique des événements courants » devient insensiblement, mais inexorablement «la chronique des images-mouvement», l'histoire du triomphe de la télévision sur toute autre forme d'histoire, ou d'Histoire.

Handke donne les raisons de l'étonnante position qu'il a choisie :

Die Frage nach der Lust zu einem Fernsehfilm traf mich zu einer Zeit, als ich gerade anfing, von Dingen und meinen Gefühlen lieber eine Geschichte zu erzählen, als, indem ich über sie schrieb, zu tun, wie wenn ich beides, Dinge und Gefühle abhandeln könnte; das Schema von Kultur kritik, mit dem man dabei umgehen mußte, erschien mir immer mehr als ein bloßes Ritual. So sagte ich also, daß ich gerne einen Fernsehfilm schreiben würde. Er sollte vom Fernsehen und von meinem Fernsehen handeln, es aber nicht abhandeln ${ }^{2}$.

4 La distance nécessaire ne va donc pas être produite de l'extérieur, par la mise en perspective critique, mais de l'intérieur, par la superposition de plusieurs niveaux narratifs; le premier est celui de la succession ordinaire des images télévisées: nouvelles, jeux, feuilletons, émissions éducatives, débats politiques, films policiers, etc. Le second forme une sorte de contre-point au premier: c'est l'irruption brutale d'images autres, des images, dit Handke, «de ses sensations, de ses propres désirs, de ses angoisses ». Ce sont des plans d'une poésie étrange, qui, comme ceux des films de Wim Wenders, ouvrent brusquement une autre dimension du regard: des trains qui traversent une plaine à la nuit tombante, des visages, des expressions, le sourire tout à coup authentique d'un comédien. Le troisième niveau narratif est celui de l'histoire des deux héros, mais qui est comme empêchée, comme si elle retombait sans cesse dans l'incroyable fouillis des images télévisées. Elle n'est pas vraiment racontée pour ellemême, elle est d'emblée intégrée dans l'histoire des événements inouïs qui renversent l'ordre social. Une des premières séquences donne le ton: on y voit une voiture s'approcher lentement du portail d'une propriété, il fait à peine jour, sur l'image apparaît le texte suivant :

1969. Alles ist im Umbruch begriffen. Kein Wert mehr wird als gesichert betrachtet, keine Ordnung mehr gilt als endgültig. Alle Vorstellungen von Gut und Böse, Recht und Unrecht, Wahr und Unwahr sind über den Haufen geworfen. Keiner mehr ist seiner Sache sicher. Eine heilsame Verwirrung hat überall eingesetzt und jedermann nachdenklich gemacht. Verstört beginnt man allerorten zu fragen, wie man denn leben solle. Das Problem, wie man die Verhältnisse zueinander neu ordnen könne, geht an niemand vorbei; es beschäftigt die Menschen in den Betrieben, in den Büros, in den Warenhäusern: kaum einer von ihnen kann sich der Uberzeugungskraft der neuen Ideen entziehen! ${ }^{3}$

5 La recherche d'un nouvel ordre social, d'un nouveau sens de l'être-ensemble, est d'emblée rapprochée par Handke du désir individuel d'une nouvelle vie. Avant même que les héros qui vont se lancer dans cette aventure n'apparaissent sur l'écran, un long plan d'ensemble fixe sur une ville en plongée est accompagné de la voix off qui dit :

Eines Tages trocknet man sich die Hände ab und sieht am Spiegel eine Ansichtskarte stecken. Man tritt näher: eine Stadt. Eine Stadt! Warum ist das einem nicht früher aufgefallen? Man ist wie im Fieber. Man zieht sich aus bis aufs Unterhemd und wäscht sich kalt. Man zittert vor Gier, als man zum Schrank geht und sich ein frisches Hemd anzieht. Jemand sagt was zu einem, aber man ist nicht mehr ansprechbar. Man ist nicht mehr ansprechbar! In die Stadt! In die Stadt gehen! ${ }^{4}$.

6 La Subversion de l'ordre et le désir utopique d'une existence autre sont de même nature et de même force. Or ces deux utopies, ou plutôt ces deux énergies, vont se 
trouver prises dans le flux des images télévisées. L'émancipation politique et l'aventure individuelle se disent d'abord hors-image, dans la voix-off qui la double, ou l'ignore : en surimpression. Bientôt le désir va réintégrer le plan de l'image, s'y fondre, se confondre avec elle : des événements courants, il ne restera plus que le cours des images, et Spade et Beaumont vont devenir les personnages fantomatiques qui les hantent, vidés de leur substance, simples exécutants de tristes pantomimes. C'est cet événement-là que raconte le film de Handke, celle de la subversion d'un désir subversif, celle de sa récupération par l'ordre télévisuel.

7 Voilà pourquoi Rainer Nägele peut affirmer :

Erzählt wird weniger die Geschichte zweier Leute, als vielmehr die Geschichte von Bildern, in denen diese Leute sich bewegen, und von denen sie bewegt werden ${ }^{5}$.

8 La déstabilisation de l'économie et des rapports sociaux, la véritable crise des valeurs qui semblait s'être emparée de tout un chacun et dans laquelle désir collectif et désir individuel se rejoignaient, au bout d'à peine quelques séquences n'est plus montrée que comme une perturbation, un dysfonctionnement. L'une des protagonistes remarque que les distributeurs automatiques, en ville, sont déjà tous vides, on n'y trouve plus que quelques bouquets de fleurs fanées. A l'exigence d'un ordre nouveau s'est insensiblement substitué l'exigence de l'ordre. Celui-ci s'est annoncé dès la huitième séquence, qui se donne comme la présentation d'un débat télévisé. La caméra se pose tour à tour sur chaque participant, un speaker récite de monotones curriculum-vitae :

John A. McCone, 1935/38 Studium in Heidelberg und Innsbruck / seit 1945 Direktor der Joshua Hendy-Eisenwerke; Direktor der Bank of America und der PazifikLebensversicherung / 1958/61 Präsident der Atom-Energie-Kommission / 1961/65 Direktor des CIA... Pr. Dr Joseph Albert Russell, 1931/34 Studium in Tübingen und Marburg / 1937/41 Dozent für Geographie / seit 1948 geographischer Berater des Leiters für Marktforschung in der Ford Motor Compagny... Robert Strange Mc Namara, 1940/43 Professor für Verwaltungskunde / 1946/61 Direktor der Ford Motor Company / 1961/68 Staatssekretär für Verteidigung / seit 1968 Präsident der Weltbank...6.

9 Or ces politiciens, pour une part empruntés à l'actualité américaine (d'ailleurs toute l'ambiance de la "ville » est très américanisée), vont apparaître sur l'écran dans les situations les plus diverses : déguisés en journalistes qui posent de fausses questions et obtiennent les réponses qu'ils désirent; interviewés chez eux, dans une intimité truquée et mise en scène; animateurs de jeux télévisés; hommes politiques qui présentent leurs programmes. Cette ubiquité est fictive, comme est fictive la conjuration du capital, de l'industrie, de la finance et de la politique. Et pourtant ni l'une ni l'autre ne surprennent le spectateur, comme si la collusion était naturelle. Bientôt Spade et Beaumont vont devenir les valets des puissants, et on les verra trottiner derrière eux, portant leurs mitraillettes ou leurs cannes de golf. Une protagoniste essaie certes de supprimer l'un d'entre eux, en lui assénant, dans le salon particulier d'un restaurant, de grands coups de bouteille de ketchup sur la tête ! Mais la révolte reste sans effet, dérisoire et drôle. Le triomphe demeure celui des grands prêtres de la communication, à la fois omniprésents et insaisissables. C'est eux qui dominent la machine aux images et c'est eux qui orchestrent la machination.

10 Ce n'est donc pas sans humour que Handke indique dans quelle direction va le cours des événements. La télévision est ici non seulement l'instrument de la manipulation des masses, mais encore celui de l'écrasement de toute révolte individuelle. Handke fait dire à ses deux grands manipulateurs en quoi consiste la «tâche» du médium, qui est de 
rabattre, d'intégrer et de totaliser l'ensemble des énergies, fussent-elles les plus intimes, comme celle du rêve et de la folie. Mac Namara dit à Mr Mann:

Unsere Aufgabe, Mr Mann, ist es, dafür zu sorgen, daß Träume und Schizophrenie zu gesellschaftlichem Bewußtsein werden und damit als Träume und Schizophrenie unnötig werden?.

11 Ce n'est pas là pourtant que réside vraiment l'intérêt du film de Handke, bien que la force de la dénonciation soit évidente. Cette dénonciation, et voilà ce qui fait ici sa véritable force, emprunte les moyens mêmes de son objet : c'est en jouant le jeu de la télévision, en se pliant à ses règles, que Handke va montrer en quoi consiste ce jeu et quelles en sont les règles. En d'autres termes, il ne va pas prouver que le petit écran devient un instrument du pouvoir, mais au contraire qu'il est, de prime abord, le pouvoir. Car les puissants, Robert Strange Mc Namara, Mr Mann, au fond, ne sont que des marionnettes, de toutes façons interchangeables. Ils ne détiennent pas le pouvoir, même s'ils s'en servent, car le pouvoir est entièrement passé du message au medium. Ce qu'il importe à Handke de mettre à jour, ce ne sont pas les rouages d'un système de domination politique, mais davantage et avant tout la domination du système compliqué des images télévisées.

12 Pourquoi ont-elles tant de pouvoir? Toute image ne l'a pas. L'image du cinéma ne se subordonne pas son spectateur, qui y est pourtant livré sans défense, dans l'obscurité des salles noires. L'image de la peinture non plus, qui cependant peut subjuguer, et comme le disaient les anciens philosophes, comme la poésie entraîner dans un monde d'illusion. L'image télévisuelle a sur tous les autres types d'images un avantage essentiel: elle n'est pas vraiment représentation, mais se donne immédiatement comme tranche du réel, tranche de vie. L'image photographique était déjà la reproduction « immédiate » de la lumière, par son inscription chimique sur le support. L'image-mouvement du cinéma y ajoutait l'illusion de la vie; l'image vidéo est le prélèvement direct d'un morceau de vie. Le petit écran permet d'assister "en valeur réelle et en temps réel » à un événement sans que la présence physique ne soit plus nécessaire : il est une sorte d'instrument d'optique, prolongement de l'œil, lunette, lucarne, fenêtre. Image non définie qui exige de l'œil un travail actif, ce médium est celui d'une participation effective, mais dans une dimension, qui, tout en se donnant pour le réel, se substitue à lui. Il y a dans le phénomène télévisuel une ambiguïté fondamentale : tout en prétendant être immédiate inscription des choses sur l'écran, l'image reste image, c'est-à-dire cadrage et choix, modèle et ordre. Rien ne déborde de l'écran, tout y trouve sa place, car le vivant y a été soumis au modèle (présumé) du vivant. Voilà pourquoi il faut reconnaître avec Baudrillard qu'il ne s'agit plus ici d'un monde de l'artifice (comme celui de la peinture), ni même d'un monde de la représentation (comme celui de la photographie et du cinéma), mais d'un monde où se produit une "précession des modèles ", et où a lieu la simulation; l'image-vidéo est dans ce sens l'image-simulacre :

Il ne s'agit plus d'imitation, ni de redoublement, ni même de parodie. Il s'agit d'une substitution au réel des signes du réel, c'est-à-dire d'une opération de dissuasion de tout processus réel par son double opératoire, machine signalétique met astable, programmatique, impeccable, qui offre tous les signes du réel et en court-circuite toutes les péripéties. Plus jamais le réel n'aura l'occasion de se produire... ${ }^{8}$.

13 L'univers du simulacre ne reflète pas le réel, mais le remplace. Et c'est ainsi qu'il a raison de lui. Ce n'est pas parce que la télévision contrecarre le désir d'émancipation, mais parce qu'elle l'intègre et fait de la révolte un événement télévisuel, que celle-ci 
« n'a pas l'occasion de se produire ». L'hyper-réalité n'est rien d'autre que ce passage à une ère où réel, vérité ou désir n'ont plus besoin de fonctionner comme référents. La perte de la troisième dimension, c'est aussi celle de la dimension de l'Histoire, ou des histoires, qui viennent toutes s'écraser dans la frontalité de l'espace signalétique du petit écran.

Ce schéma capital de frontalité et d'écran est thématisé systématiquement dans le film de Handke. L'écran est partout : celui du parcmètre pris en gros-plan, compteur de taxi, immense horloge pointeuse, panneau des départs d'avions dans l'aéroport, horloge de la télévision, monstrueuse et terrifiante, qui scande le temps « réel », celui qui rythme les émissions. Tous ces écrans font partie, comme le remarque par ailleurs Paul Virilio ${ }^{9}$ d'un vaste réseau "de détection et de contrôle", où le signe est d'emblée un indice. L'espace de communication, qui a remplacé l'espace de représentation n'est rien d'autre que cette présence obsédante de l'écran devant lequel on n'est plus que le récepteur de messages. Archétype de la perception policière, la vignette, le cadre, l'écran induit un rapport au monde qui n'est plus celui de la communion, mais celui de la surveillance. Dès lors, tout spectacle devient détection, comme Spade et Beaumont, embusqués derrière une fenêtre et regardant dans une rue banale, en font l'expérience :

daß man, je länger man hinunter schaut, nur noch Signale sieht, und prüft, ob alles, was man sieht, nach diesen Signalen funktioniert: jetzt springt die Ampel auf Rot, und wer läuft noch über die Straße?... Und was bedeutet es, daß dort die Jalousie heruntergelassen wird, am hellichten Tag? Und geht jemand durch die Hintertür in das Geschäft? Und was für besondere Kennzeichen haben die Leute, die ins Juweliergeschäft gehen! (Lange Pause) - Man kommt sich wie ein Polizist vor, wenn man so hinunter schaut ${ }^{10}$.

La seconde caractéristique de l'univers des signes est celui de la circularité de l'information, de l'échange, des flux. L'ordre social n'est pas la délégation d'une liberté concédée pour la sécurité communautaire, et reprise lorsqu'elle ne répond plus à cette exigence. Cette conception prévalait encore dans le désir d'un " autre être ensemble » que le début du film disait sans ambiguïté. L'ordre social ne se modifie pas selon ce désir, qui est celui d'un individu-citoyen raisonnable et responsable (voilà pourquoi émancipation sociale et émancipation individuelle sont d'abord assimilables, et montrées dans le même geste par Handke). L'ordre social qui n'est plus celui de la polis mais celui du petit écran, qui est la seule forme réelle d'espace public, intègre le désir comme élément de fonctionnement de son système, et la révolte comme correcteur de sa trajectoire : il les gère. Handke montre que la chronique des événements courants, est la chronique des énergies, de la circulation des flux et des échanges dans une communauté qui se conçoit sur le modèle de ce qu'il appelle « la ville». Jean-François Lyotard la nomme la mégapole, et ce qu'il en affirme ressemble étrangement à ce que Handke en a perçu :

Cette forclusion du désir et l'abaissement de tous les signes dans l'imaginaire de la gestion donnent à la mégapole son style étrange, celui d'une zone à la fois précaire et confortable ${ }^{11}$.

Il y a un certain confort dans la circularité des échanges qui ne se font plus que sous l'espèce du simple échange marchand, «le seul qui fonctionne ", dit cyniquement l'un des personnages ${ }^{12}$. Tous les autres sont interrompus et Handke multiplie les plans de téléphones qui ne sonnent plus, de sonnettes qui n'appellent plus personne, de discussions politiques truquées, d'échanges amoureux ratés et de pseudo-débats qui ne 
débouchent que sur la forclusion de leur propre logique. Car toute une dimension de l'échange symbolique a été ici perdue, ou niée, remplacée par la simple circulation de messages d'un pôle à l'autre, par un simple flux d'informations codées.

Dans L'Angoisse du gardien de but au moment du pénalty, qui date des mêmes années, Handke met en scène le phénomène d'un réel totalement vécu sous la modalité des images: et c'est l'entrée dans la schizophrénie de Joseph Bloch, qui n'a d'autre existence que d'être le gardien de but, face au terrain, métaphore du récepteur des messages et des stimuli face à la frontalité de l'univers des signes. La Chronique des événements courants fait les mêmes observations, sans leur donner leur signification psychologique. Elle reste laconique comme un constat.

Au-delà de cette simple observation d'un phénomène qui n'est pas marginal, mais central pour la compréhension de notre vie sociale, il y a dans le film de Handke toute une analyse de l'image télévisée, "vue de l'intérieur ». Handke développe deux caractéristiques de celle-ci, qui constituent un paradoxe apparent : l'image-vidéo du petit écran est à la fois discontinue et prise dans un continuum. L'image télévisuelle ne prétend pas être image totale, elle n'est jamais qu'un bout, un fragment de la totalité, n'ayant même pas valeur de symbole de celle-ci : reportages, scènes live, témoignages, déclarations, tout ceci ne veut nullement avoir d'autre valeur que la sienne propre. Ainsi Handke surnote-t-il le fragment, appuie-t-il sur le précaire de l'image : il n'y a dans son film aucun "plan parfait » aucune image qui soit totalement satisfaisante. Il existe un décalage entre le son et l'image, ou entre l'énoncé et celle-ci, quelque chose d'irritant et de frustrant que l'on connaît de l'esthétique de "l'image sale » revendiquée par Godard. Le montage du film ajoute à cette impression, Handke l'a voulu délibérément brutal. Mais, en même temps, dans le film tout est fait pour que l'on ait le sentiment qu'on vient de passer l'antenne, que l'émission en suit une autre, que les images se succèdent en une suite interrompue et obsédante. Le petit écran a horreur du vide, et c'est d'ailleurs la raison pour laquelle Wim Wenders le filme si souvent vide, inutile, ou plutôt mis hors d'état de nuire. Handke a compris que l'image télévisée ne vit pas de son contenu, ni même de son intensité, mais de sa continuité. Il y a dans son film tout un travail sur cette continuité, nécessaire à la permanence de l'univers des signes. Un plan saisissant l'illustre mieux que tout autre: on y voit d'abord en vue fixe une fontaine éclairée la nuit, quelque chose qui pourrait être l'emblème d'une chaîne de télévision, puis nos deux héros, Beaumont et Spade «entrent dans l'image ", intégrant l'histoire de leur désir dans le réseau des images-vidéo, et passant entièrement en elles. Il n'y a plus dès lors d'autre histoire à écrire que l'histoire des images $^{13}$.

Ce n'est pas sans une certaine ironie mélancolique que Handke clôt son film, en une séquence qui se lit comme un adieu à l'utopie :

Die Fernsehuhr, 25 Sekunden vor 20 Uhr. Der Sekundenzeiger läuft. Als er auf acht ist: Gongschlag. Der Sprecher erscheint im Bild und redet: «Guten Abend, meine Damen und Herren. Den gemeinsamen Bemühungen aller Beteiligten ist gelungen, was kaum jemand mehr für möglich gehalten hätte: der lang andauernde, hartnäckige...» Der Ton, schon zu Beginn des Satzes leise, ist immer leiser geworden, jetzt ist er unhörbar. Man sieht den Sprecher ein bißchen weiterreden, dann - langsam wird das Bild ausgeblendet - setzt Musik ein, die während des abschließenden Rolltitels andauert Eric Burdon «When I Was Young» ${ }^{14}$. 


\section{NOTES}

1. 1 - Heiko Blum, Gespräch mit Peter Handke, in M. Scharang (Hrg): Uber Peter Handke, Suhrkamp, Francfort, 1972, p. 80.

2. - Peter Handke: Nachwort zu Chronik der laufenden Ereignisse, Suhrkamp, Francfort, 1971, p. 128.

3. - Chronik der laufenden Ereignisse, p. 9.

4. - Ibidem, p. 14.

5. - Rainer Nägele et Beate Voris : Peter Handke, Verlag Autorenbücher, Munich, 1978, p. 102.

6. - Chronik der laufenden Ereignisse, p. 32.

7. - Ibidem, p. 48.

8. - Jean Baudrillard, Simulacres et simulation, Galilée, Paris, 1981, p. 12.

9. - Paul Virilio : La machine de vision, Galilée, Paris, 1988.

10. - Chronik der laufenden Ereignisse, p. 54.

11. - Jean-François Lyotard : Moralités post-modernes, Galilée, Paris, 1993, p. 36.

12. - Voir séquence 23.

13. - Voir séquence 9.

14. - Chronik der laufenden Ereignisse, p. 122.

\section{RÉSUMÉS}

La Chronique des événements courants est avant tout une chronique des images. Mêlant diégèse et critique, par la subtile superposition de différents plans narratifs, Handke décrit le triomphe de l'image télévisée qui vient à bout de toute histoire, et de l'Histoire. Car cette image-là n'est pas tant instrument du pouvoir que pouvoir à elle seule : simulacre, elle ne reproduit pas le réel, comme le note Baudrillard, mais le remplace. C'est en faisant fonctionner le médium, en entrant dans son jeu, que Handke en montre les règles. Et il dresse ainsi le laconique constat de la domination d'un univers de signes dans lequel tout s'abîme, désir individuel comme utopie collective.

Handkes Fernsehfilm ist halb erzählte Geschichte und halb Lehrfilm über das Funktionieren des Fernsehens überhaupt, sodaß die Chronik der laufenden Ereignisse unversehens zu einer Chronik der laufenden Bilder wird. Erzählt wird der Sieg des Fernsehens über jede andere Art von Geschichte. Handke zeigt nicht, wie hier das Medium zum Instrument der Macht wird, sondern vielmehr wie es von vornherein die Macht ist: Das Fernsehbild ist ein Simulakrum und als solches - so Baudrillard - kann es die Realität nicht nur ersetzen, sondern auch erzeugen. Handkes Kritik bedient sich der Ausdrucksmittel des Mediums selbst und kommt damit dem Prozeß der Wahrnehmung im Fernsehen nahe. Lakonisch wird festgestellt, wie ein Netz von Zeichen entsteht, das letztendlich jeden Austausch unmöglich und jede Utopie unnütz macht. 
AUTEUR

ARLETTE CAMION

Université d'Orléans 